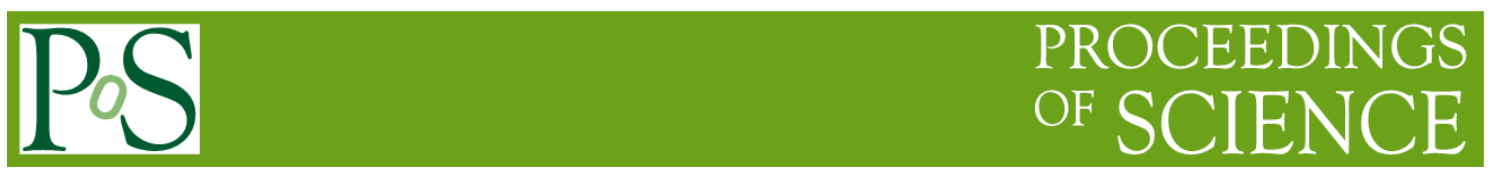

\title{
Uncertainty Evaluation Approach for Cognitive Network
}

\author{
Sainan Liu ${ }^{12}$ \\ School of electronic Information, Nan tong University, Nan tong Jiangsu, 226019, China \\ Email: I iusainan10126. com
}

\section{Zhenguo Shi}

School of Computer Science and Technology, Nantong University, Nantong, Jiangsu, 226029, China Email: $1316838447 @ q q$. com

The cognitive network has become a hot issue in the study of next generation network and its network performance has also been given more and more attentions. Now as to the cognitive network performance evaluation, the study is to consider some performance index in isolation, failing to consider various performance indicators in various kinds of uncertainty factors in the process of evaluation. In order to solve the existing performance evaluation of cognitive network with random and fuzzy problems, a method for evaluating the uncertainty of cognitive network is proposed in this paper. The paper studies and then puts forward the cognitive network performance evaluation method based on cloud model, which implements quantitative and qualitative swaps of cognitive network performance. Finally, the experiment is given to demonstrate the feasibility of the evaluation method.

CENet2015

12-13 September 2015

Shanghai, China

\footnotetext{
${ }^{1}$ Speaker

${ }^{2}$ Correspongding Author
} 


\section{Introduction}

Cognitive network [1] can adjust the internal communication network configuration at real-time and intelligently adapt to changes in the external environment through the external environment perception. This type of network is a network of end-to-end QoS (Quality of Service) performance improvement as the goal while focusing on the entire network instead of some elements. Because of the unique nature, the cognitive network has become a hot spot of research both at home and abroad [2 4]. It's these features that are beneficial of providing users with a more suitable QoS guarantee.

In order to make the cognitive network provide users with better service quality, how to effectively know the network service quality evaluation is very important. Scholar Chen Xi put forward a method that evaluated the performance of the cognitive network from backlog and time delay by depicting a type of new network data arrival feature and channel characteristic of service [5]. Dr.Contains proposed a cognitive network management model, which introduced the cognitive measurement system to continuously monitor and diagnose the potential problem of network performance. [6]. Scholar Fen Guangshen et al.put forward a method of ensuring the users' QoS level by using utility function, interrupt management mechanism and active characteristics of cognitive network [7] .

Currently, the network performance evaluations about cognitive network are mostly in isolation to consider some performance index while failing to consider the uncertainty of each performance in the evaluation process. The indicators use different evaluation approaches. Some can only use natural language and others can use accurate numeric; therefore, how to deal with the uncertainty in the evaluation process and then accurately evaluate the cognitive network performance become the key to the study. In order to solve the above problems, on the cognitive network of business transmission as the research background on the basis the relevant theories of cloud model [8], this paper proposes an uncertainty method of evaluation for the cognitive network, which can effectively solve the randomness and fuzziness of the indicators in the evaluation process.

\section{Evaluation Model}

\subsection{Cloud Model}

\subsubsection{Basic Concept of the Cloud}

The cloud model is a kind of uncertainty intelligent transformation model in the field of artificial intelligence, which mainly reflects on the concept of uncertainty, fuzziness and randomness. It is used to describe three numerical characteristics, namely, the expectation Ex, the entropy En and the hyper entropy He. The expected value of Ex is the concept of the standard value and it reflects the quality concept corresponding information centre value; the entropy En is the measurement of the uncertainty degree of qualitative concept. The larger the entropy is, the vaguer concept will be; the hyper entropy is the entropy of the entropy, reflecting the degree of dispersion of cloud, that is, to determine the degree of uncertainty [8].

\subsubsection{Cloud Generator}

Normal cloud generator and inverse cloud generator are the two important algorithms in cloud model [9]. Normal cloud generator can realize the qualitative concept to the quantitative, that is, the digital characteristics of cloud can produce the cloud droplets while the inverse cloud generator is the reverse process.

As to the normal cloud generator algorithm:

Input: qualitative concept of expectation Ex, entropy En and hyper entropy $\mathrm{He}$ 
Output: quantitative location of the cloud droplets in the data space the concept of uncertainty represented by each cloud droplet

(1) generator a random number $E n^{\prime}$ with En as the mean and $\mathrm{He}^{2}$ as the variance;

(2) generator a random number $\mathrm{x}$ with $\mathrm{Ex}$ as the mean and $E n^{\prime 2}$ as the variance;

(3) calculate $y=e^{-(x-E x)^{2} / 2\left(E n^{\prime}\right) 2}$;

(4) make (x, y) as a cloud droplet in the theory domain;

(5) repeat the above steps until the specified cloud droplets.

Inverse cloud generator algorithm:

Input: $\mathrm{N}$ number of cloud droplets

Output: the digital characteristics of the cloud (expectation Ex, entropy En and hyper entropy $\mathrm{He}$ )

(1) calculate the sample mean ${ }_{x}^{-}$and the sample variance $s^{2}$ according to the cloud droplet $x_{i}$;

(2) calculate the expectation as $\mathrm{Ex}=\overline{\boldsymbol{x}}$;

(3) calculate the entropy as $\mathrm{En}=\sqrt{\frac{\pi}{2}} \cdot \frac{1}{n} \sum_{i=1}^{n}\left|x_{i}-E x\right|$;

(4) calculate hyper entropy as $\mathrm{He}=\sqrt{s^{2}-E n^{2}}$.

\subsection{Cognitive Network Performance Evaluation Model based on Cloud Model}

The performance evaluation of the cognitive network is the first to measure the multiple basic performance indicators and then comprehensively analyze the multiple performance indicators to obtain the current network's overall operation. The concentration of certain performance targets mutating will affect the performance of the network and the network state can optimize the performance if multiple.

In this article, the local perception of cognitive users and the QoS parameter information acquired by domain nodes will report to their respective domain cognitive server. Then, each cognitive domain server will report the information to the intelligent evaluation module to obtain final assessment of network performance. Under the actual situation of network running in cognitive network in this paper, with cloud model theory, on the basis of study of the comprehensive evaluation of each base indicator for the operation situation of network as a whole, the fuzziness and randomness of the network evaluation process can be reflected objectively. The evaluation model is shown in Fig. 1. In order to better evaluate the overall performance of the network, we can specify the index weight in advance according to the specific situation. This article by using fuzzy analytic hierarchy process to determine the weight of each index of size [10]. In this evaluation model, each sample points of sampling for cognitive network as cloud droplets will be sent to the inverse cloud generator to get their own digital characteristics of the normal cloud, which can achieve a qualitative evaluation of the sample data. Finally, the qualitative evaluation of these values will be expressed by normal cloud.

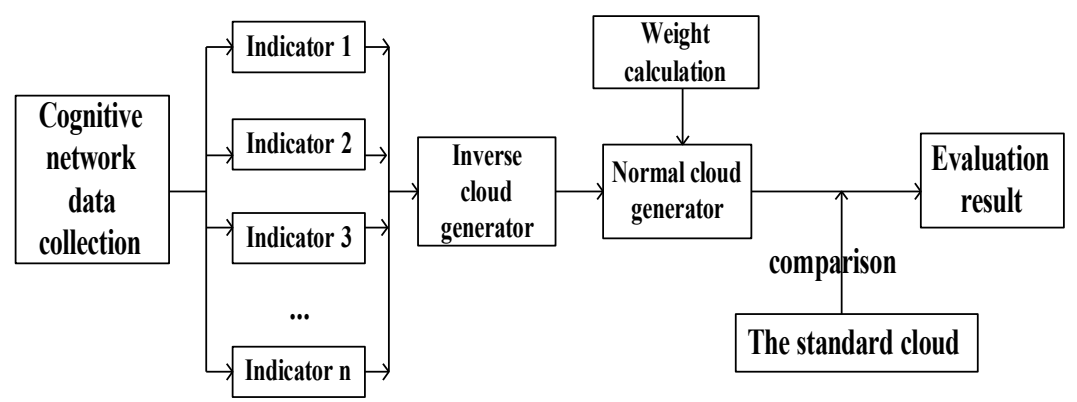

Figure 1: Evaluation Model of Cognitive Network Performance

\section{Evaluation Algorithm}


The performance evaluations of the specific steps are shown as follows:

Input: information from all data collection points of cognitive network, $X=\left\{x_{1}, x_{2}, \ldots, x_{n}\right\}$

Output: comprehensive evaluation results of cognitive network performance

Step1: collecting QoS information and building indicators domain of cognitive network

$X=\left\{\left\{x_{11}, x_{12}, \ldots, x_{1 k}\right\},\left\{x_{21}, x_{22}, \ldots, x_{2 k}\right\}, . .,\left\{x_{n 1}, x_{n 2}, \ldots, x_{n k}\right\}\right\}$

Step2: establish the evaluation theory field $Y=\left\{y_{1}, y_{2}, \ldots, y_{m}\right\}$

Step3: use FAHP to calculate the corresponding index weights $W=\left\{w_{1}, w_{2}, \ldots, w_{n}\right\}$

Step4: use the cloud model to calculate the membership degree of theory field $\mathrm{X}$ to the comments field $\mathrm{Y}$ and comparing with the standard cloud

\section{Experimental Verification and Analysis}

\subsection{Cognitive Network Experimental Environment}

The article uses network simulation tool NS2 to build cognitive network platform. The topology structure is shown in Fig. 2. In the figure, there are three cognitive domains, including 12 leaf nodes representing the host and 3 non-leaf nodes representing the domain server. The experimental simulation uses data transmission business as an example. We select the time interval $\mathrm{T}$ to network operation results and then a plurality of sample points can be obtained. The parameter of the sample points obtained from each cognitive domain is shown in Table1. The form of the result is a parameter of triples (delay $/ \mathrm{ms}$, jitter $/ \mathrm{ms}$, packet loss rate). Time 9 to time 12 is the overload condition of route 1 to route 2 .

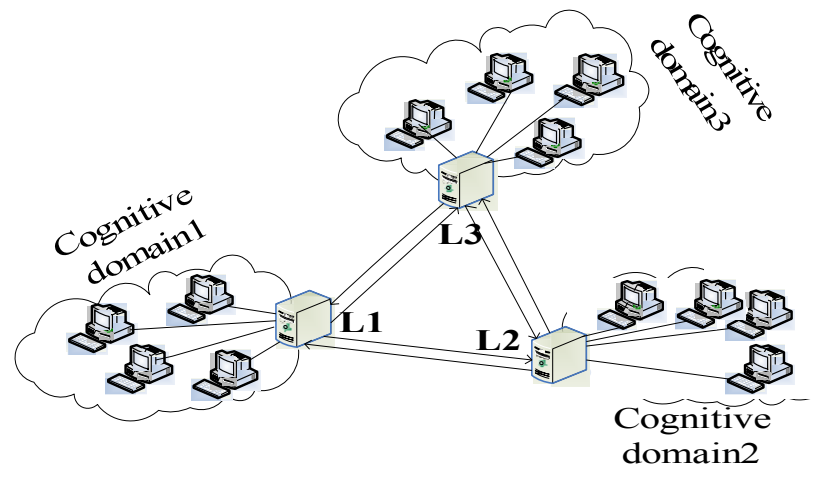

Figure 2: Network Topology

\begin{tabular}{|l|l|l|l|}
\hline Time & Cognitive domain1 & Cognitive domain2 & Cognitive domain3 \\
\hline T1 & $(18.21,0.43,0.08)$ & $(20.31,0.55,0.12)$ & $(14.06,0.28,0.01)$ \\
\hline T2 & $(18.44,0.47,0.09)$ & $(20.61,0.75,0.14)$ & $(14.79,0.29,0.03)$ \\
\hline T3 & $(19.67,0.52,0.09)$ & $(20.93,0.88,0.16)$ & $(15.26,0.34,0.04)$ \\
\hline T4 & $(21.46,0.57,0.11)$ & $(22.71,0.95,0.17)$ & $(15.84,0.41,0.04)$ \\
\hline T5 & $(23.21,0.62,0.12)$ & $(25.35,0.92,0.15)$ & $(18.27,0.49,0.05)$ \\
\hline T6 & $(26.34,0.64,0.11)$ & $(28.31,0.94,0.16)$ & $(21.19,0.48,0.06)$ \\
\hline T7 & $(27.66,0.65,0.11)$ & $(31.79,0.96,0.18)$ & $(22.07,0.51,0.05)$ \\
\hline T8 & $(29.84,0.67,0.13)$ & $(32.31,0.95,0.17)$ & $(23.24,0.49,0.04)$ \\
\hline T9 & $(50.72,1.64,0.87)$ & $(52.37,1.75,0.89)$ & $(24.37,0.52,0.06)$ \\
\hline T10 & $(53.76,1.68,0.89)$ & $(54.72,1.76,0.91)$ & $(25.58,0.53,0.07)$ \\
\hline T11 & $(54.28,1.69,0.89)$ & $(56.68,1.75,0.93)$ & $(27.49,0.57,0.08)$ \\
\hline T12 & $(57.36,1.70,0.91)$ & $(60.49,1.79,0.96)$ & $(28.36,0.58,0.08)$ \\
\hline T13 & $(35.94,0.79,0.15)$ & $(36.38,0.85,0.16)$ & $(28.24,0.56,0.07)$ \\
\hline T14 & $(31.82,0.74,0.13)$ & $(33.85,0.82,0.14)$ & $(29.86,0.60,0.08)$ \\
\hline T15 & $(30.68,0.78,0.15)$ & $(32.62,0.84,0.16)$ & $(29.37,0.62,0.09)$ \\
\hline T16 & $(30.92,0.79,0.14)$ & $(32.48,0.86,0.18)$ & $(29.94,0.63,0.08)$ \\
\hline
\end{tabular}

Table 1:Simulation Results

4.2 Performance Evaluation 
The index weights obtained by using FAHP are shown in Fig. 3. The data samples input the evaluation system and then we can obtain the results shown in Fig. 4, which can be seen from the diagram. Generally speaking, the network performance is better. Fig. 5 shows the comparison chart of the network performance at different time and different load conditions. It can be seen that from time 1 to time 8 and from time 3 to time16, the network performance is ideal for network load which is normal; during the period from time 9 to time12, the network performance is bad because the network is overloaded. As seen from the evaluation results, the network performance put forward in this article can accurately reflect the performance of the network.

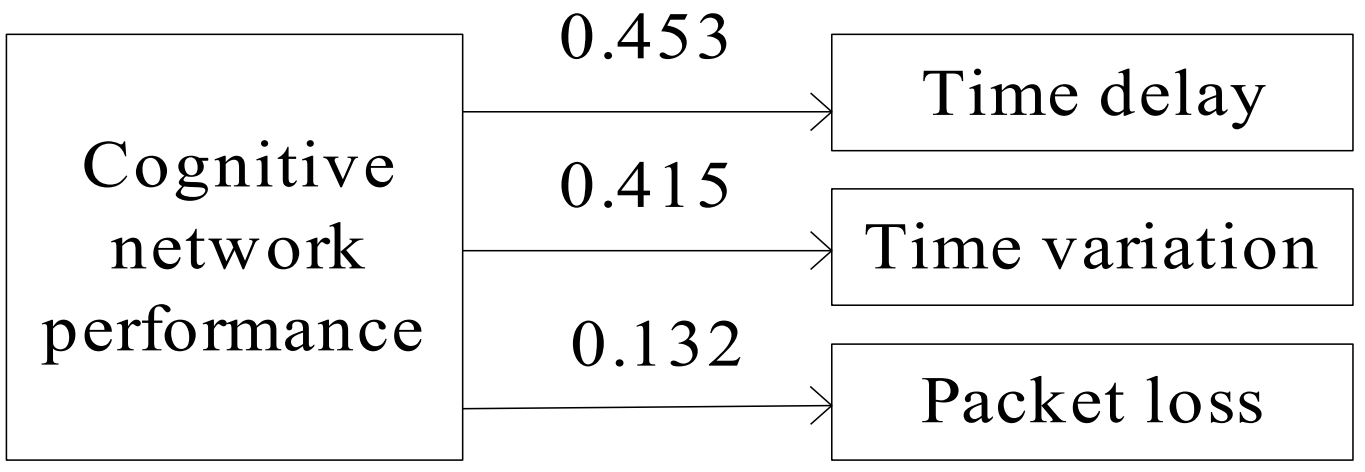

Figure3: Network Performance Index Weights

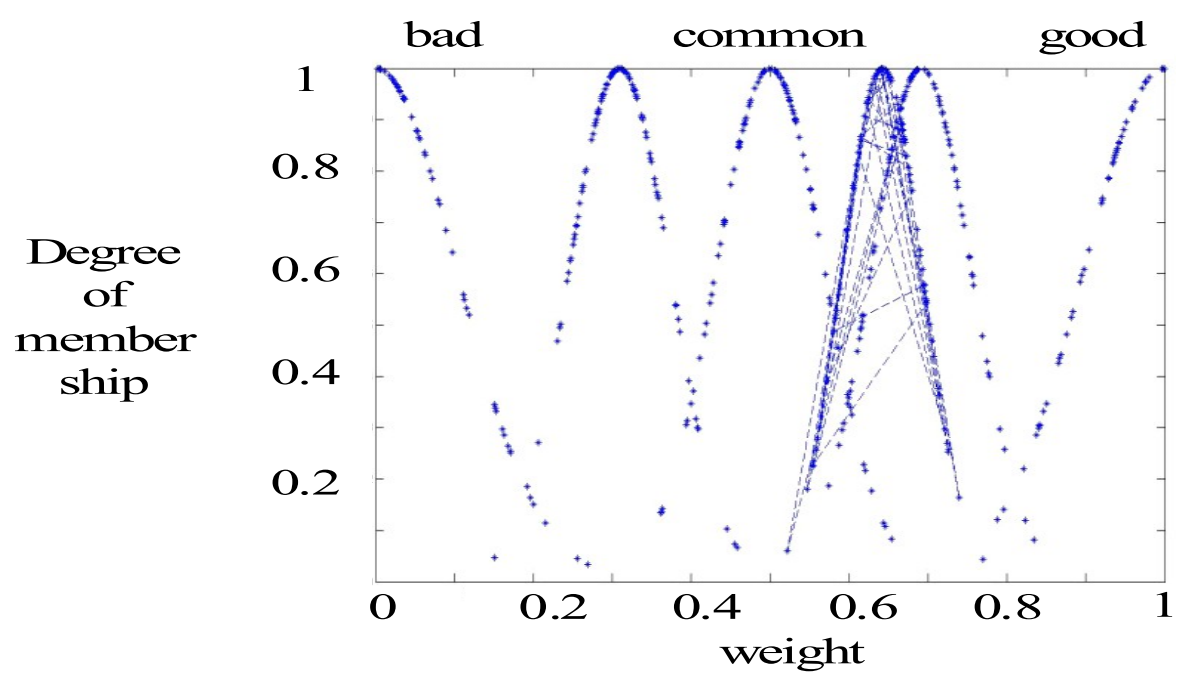

Figure 4: Comprehensive Evaluation Results 


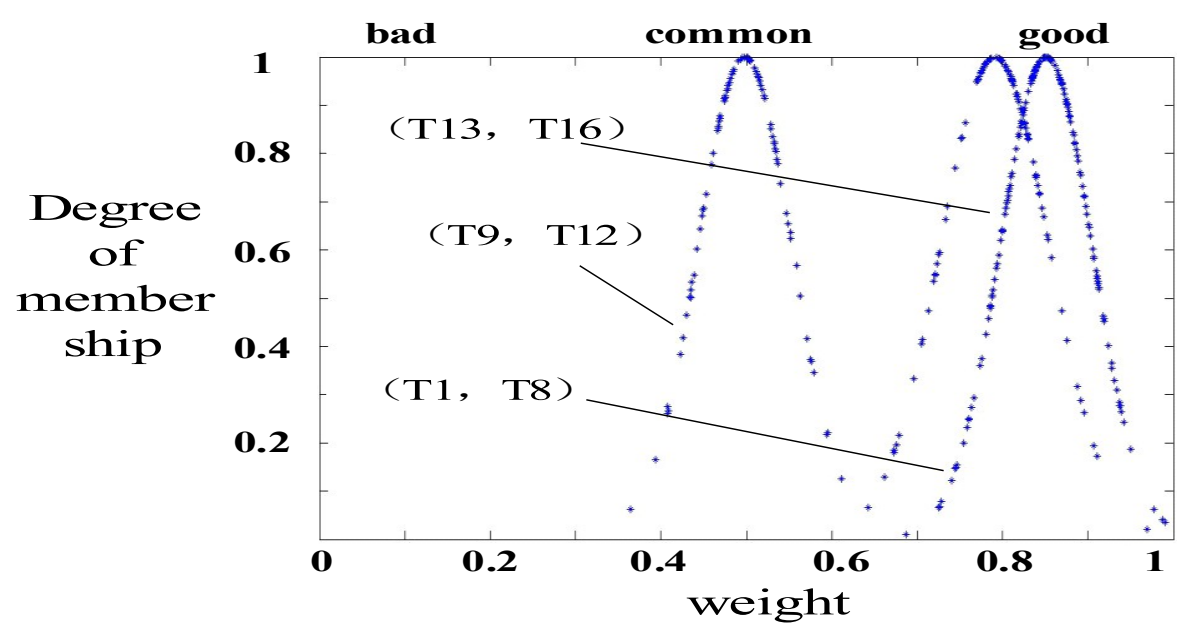

Figure 5: Comparison of Network Performance under Different Load Conditions

\section{Summary and Outlook}

Aiming at performance evaluation of cognitive network in a variety of random and fuzzy problems while in combination with the theory of cloud model and FAHP, the article evaluates the performance of cognitive network from the uncertain angle. The evaluation method uses the uncertainty and the stable tendency during the processing of the cloud droplet generation so as to realize the network performance assessment values and convert the domain of uncertainty reviews. Simulation results show the feasibility of this evaluation method. The text of the evaluation results is obtained in the simulation case. How will the performance evaluation of the cognitive network is extended to the practical application is the next step, which the research will focus on.

\section{References}

[1]R.W. Thomas, L.A. DaSilva, Cognitive Networks[C].Proc IEEE DySpan 2005, Maryland, USA, Nov, 2005:352-360

[2]G.Sakellari, Georgia. The cognitive packet network: A survey [J]. Computer Journal, 2010, 53(3):268-279

[3] A. Hey Laurence, Reduced complexity algorithm for cognitive packet network [J].Computer Communications.2008, 31(16):3822-3830

[4] D. Sarkar, Transport layer protocols for cognitive networks [A].IEEE Computer Communications Workshops. an Diego,CA,United states,2010,1-6

[5] X. Chen, T. Xu, Performance Evaluation of Cognitive Radio Networks with Parallel Channels [J],Journal of Computer Research and Development,2013,50(10),2126-2132

[6] Dr.Contains. An Introduction to the Cognitive Concept as a Cost-Effective Method for Network Performance Management[C].IEEE Wireless Communications and Networking Conference, Las Vegas, NV2008:3197-3201

[7] G. S. Fen, H. Q. Wang, Dynamic self-configuration of user QoS oriented to cognitive network[J],Journal on Communications,2004,15(11):1583-1594

[8] D.-y. Li, C.-Y. Liu, Artificial Intelligence with Uncertainty [J], Journal of Software, 2004, 15(11):1583-1594 
[9] D.-Y. Li, Y. Du, Artificial Intelligence with Uncertainty [M], National Defense Industry Press, 2005

[10] J.-J. Zhang, Fuzzy Analytic Hierarchy Process [J], Fuzzy Systems and Mathematics, 2000, 14(2):80-88 